\title{
AN EXTREMAL PROBLEM FOR THE HYPERBOLIC METRIC ON DENJOY DOMAINS
}

\author{
DIMITRIOS BETSAKOS
}

\begin{abstract}
Suppose that $\Omega$ is a domain in the extended complex plane and assume $\Omega$ contains the origin and that the boundary of $\Omega$ lies on the interval $[-1,1]$ and has total length $2 m, 0<m<1$. We study the problem of finding the infimum of the density of the hyperbolic metric $\lambda(0, \Omega)$ at the origin among all such domains $\Omega$. We show that if $m$ is sufficiently large, the infimum is attained uniquely for the doubly connected domain which is symmetric in the imaginary axis. This result improves an estimate of A.Yu. Solynin. We also show that for sufficiently small $m$ the above domain is not any more extremal.
\end{abstract}

\section{INTRODUCTION}

Let $\Omega$ be a domain in the extended complex plane $\overline{\mathbb{C}}=\mathbb{C} \cup\{\infty\}$. Suppose that $\Omega$ is a hyperbolic domain; that is, the complement $\overline{\mathbb{C}} \backslash \Omega$ of $\Omega$ contains at least three points. Then the unit disk $\mathbb{D}=\{z \in \mathbb{C}:|z|<1\}$ is a universal covering surface of $\Omega$. The density of the hyperbolic (Poincaré) metric for $\Omega$ is a function $\lambda(\cdot, \Omega): \Omega \rightarrow(0, \infty)$ defined as follows: Let $z \in \Omega \backslash\{\infty\}$ and let $F: \mathbb{D} \rightarrow \Omega$ be the holomorphic universal covering map with $F(0)=z$ and $F^{\prime}(0)>0$. Then

$$
\lambda(z, \Omega)=\frac{1}{F^{\prime}(0)} .
$$

If $\infty \in \Omega$, we consider the image $G$ of $\Omega$ under the inversion $1 / z$, and define $\lambda(\infty, \Omega)=\lambda(0, G)$.

A fundamental property of the function $\lambda(\cdot, \Omega)$ is the principle of the hyperbolic metric which implies that if $\Omega$ and $D$ are hyperbolic domains, $f: \Omega \rightarrow D$ is a holomorphic function, $z \in \Omega \cap \mathbb{C}$, and $f(z) \in D \cap \mathbb{C}$, then

$$
\lambda(f(z), D)\left|f^{\prime}(z)\right| \leq \lambda(z, \Omega), \quad z \in \Omega
$$

with equality if and only if $f$ is a covering map. If we choose $f$ to be the inclusion map, we obtain the domain monotonicity of the hyperbolic metric:

$$
\text { If } \Omega \subset D \text {, then } \lambda(z, D) \leq \lambda(z, \Omega), \quad z \in \Omega \cap \mathbb{C} \text {. }
$$

For these and other properties of the hyperbolic metric we refer to [3, Chapter VIII], [4, Chapter 9] and [7].

Date: May, 2009.

1991 Mathematics Subject Classification. 30F45, 30C75.

Key words and phrases. Hyperbolic metric, polarization, Denjoy domain, complete elliptic integral. 
The hyperbolic metric has been much studied in the context of geometric function theory because it provides information about distortion, covering, and other properties of holomorphic functions; see, for example, [2], [3], [4], [7], [11].

We shall study the function $\lambda$ when $\Omega$ is a Denjoy domain; that is, $\mathbb{C} \backslash \Omega$ is a subset of the real axis $\mathbb{R}$. We denote by $|E|$ the one-dimensional Lebesgue measure of a measurable set $E \subset \mathbb{R}$. A.Yu. Solynin [10] posed the following extremal problem: For $0<m<1$, let

$$
\Lambda(m)=\inf \{\lambda(0, \Omega): \Omega=\overline{\mathbb{C}} \backslash E, E \subset[-1,1] \backslash\{0\},|E|=2 m\} .
$$

Find $\Lambda(m)$ and the corresponding extremal domains.

Solynin proved that for $m \geq 1-\sqrt{3} / 6 \approx 0.7113$, the infimum is attained only for the domain

$$
\Omega_{2}(m):=\overline{\mathbb{C}} \backslash([-1,-1+m] \cup[1-m, 1]) .
$$

We shall prove some results on Solynin's problem.

Theorem 1. There exists a number $m^{*}, 0.31861<m^{*}<0.31862$, with the following property:

Suppose that $m^{*} \leq m_{1}<1, m^{*} \leq m_{2}<1$. If

$$
\Omega=\overline{\mathbb{C}} \backslash\left(E_{1} \cup E_{2}\right), \quad E_{1} \subset[-1,0),\left|E_{1}\right|=m_{1}, E_{2} \subset(0,1],\left|E_{2}\right|=m_{2},
$$

then

$$
\lambda(0, \Omega) \geq \lambda\left(0, \Omega_{2}\left(\frac{m_{1}+m_{2}}{2}\right)\right) .
$$

Equality holds if and only if $\Omega=\Omega_{2}\left(\frac{m_{1}+m_{2}}{2}\right)$.

The next result follows easily from Theorem 1 and is slightly stronger than the result of Solynin mentioned above.

Corollary 1. Suppose that $m \geq \frac{1+m^{*}}{2} \approx 0.6593$. Then the infimum in (1.1) is attained only for the domain $\Omega_{2}(m)$.

Solynin $[10$, p. 2273$]$ remarks that it might happen that $\Omega_{2}(m)$ is not extremal if $m$ is sufficiently small. We confirm this conjecture by studying the domains $\Omega_{2}(m)$ and

$$
\Omega_{1}(m):=\overline{\mathbb{C}} \backslash[-1,-1+2 m], \quad 0<m<\frac{1}{2} .
$$

In fact, we will compute the exact values of $\lambda\left(0, \Omega_{1}(m)\right)$ and $\lambda\left(0, \Omega_{2}(m)\right)$ in terms of complete elliptic integrals. The computation involves the universal covering mappings for these domains which we find using the Jacobi elliptic sine function.

Theorem 2. There exists a number $m^{\sharp}, 0.3684<m^{\sharp}<0.3685$, with the following properties:
(a) If $m<m^{\sharp}$, then $\lambda\left(0, \Omega_{1}(m)\right)<\lambda\left(0, \Omega_{2}(m)\right)$.
(b) If $m>m^{\sharp}$, then $\lambda\left(0, \Omega_{1}(m)\right)>\lambda\left(0, \Omega_{2}(m)\right)$.
(c) If $m=m^{\sharp}$, then $\lambda\left(0, \Omega_{1}(m)\right)=\lambda\left(0, \Omega_{2}(m)\right)$. 
For the proof of Theorem 1, we will need the following theorem which is of some independent interest.

Theorem 3. Suppose that $0<m_{1}<1,0<m_{2}<1$. If

$$
\Omega=\overline{\mathbb{C}} \backslash\left(E_{1} \cup E_{2}\right), \quad E_{1} \subset[-1,0),\left|E_{1}\right|=m_{1}, E_{2} \subset(0,1],\left|E_{2}\right|=m_{2},
$$

then

$$
\lambda(0, \Omega) \geq \lambda\left(0, \Omega^{*}\right)
$$

where $\Omega^{*}=\overline{\mathbb{C}} \backslash\left(\left[-1,-1+m_{1}\right] \cup\left[1-m_{2}, 1\right]\right)$. Equality holds if and only if $\Omega=\Omega^{*}$.

In view of the above results, it is natural to conjecture a solution of Solynin's problem:

$$
\Lambda(m)= \begin{cases}\lambda\left(0, \Omega_{1}(m)\right), & m \leq m^{\sharp}, \\ \lambda\left(0, \Omega_{2}(m)\right), & m \geq m^{\sharp} .\end{cases}
$$

Theorem 3 reduces the problem to the case when $\Omega=\Omega^{*}$, namely when $\Omega$ is doubly connected. An explicit expression for $\lambda\left(0, \Omega^{*}\right)$ can be found but it is rather complicated.

The proofs of the theorems use polarization and ordering inequalities for the hyperbolic density. These inequalities, which are due to Solynin [8], [9], are reviewed in the next section.

\section{TOOLS}

2.1. Reflection in a circle. We will use reflections with respect to circles centered on the real axis. Let $C=C\left(x_{o}, r\right)$ be such a circle (center $x_{o}$, radius $r$ ). The reflection of a point $z \in \mathbb{C}$ with respect to $C$ is the point

$$
\mathcal{R}_{C} z:=x_{o}+\frac{r^{2}}{z-x_{o}} .
$$

We also set $\mathcal{R}_{C} \infty=x_{o}$ and $\mathcal{R}_{C} x_{o}=\infty$.

2.2. Polarization with respect to a circle. We review here only the aspects of the theory of polarization that we will need later. For more information about polarization, we refer to [8], [11] and the references therein.

Let $C=C\left(x_{o}, r\right)$ be a circle in $\mathbb{C}$. Let $E$ be any set in $\overline{\mathbb{C}}$. We divide $E$ into three disjoint sets $S, U, V$ as follows:

$S=E \cap \mathcal{R}_{C} E \quad$ (the symmetric part of $\left.E\right)$.

$U=\left(E \cap\left\{\left|z-x_{o}\right|<r\right\}\right) \backslash S \quad$ (the inner non-symmetric part of $E$ ).

$V=\left(E \cap\left\{\left|z-x_{o}\right|>r\right\}\right) \backslash S$ (the outer non-symmetric part of $E$ ).

The polarization $\mathcal{P}_{C} E$ of $E$ with respect to the circle $C$ is the set

$$
\mathcal{P}_{C} E=S \cup U \cup \mathcal{R}_{C} V .
$$


It is clear that the polarization of an open set is open. In general, the polarization of a domain is not a domain. However, the polarization of a Denjoy domain is always a Denjoy domain.

Suppose that $\Omega$ is a Denjoy domain with $0 \in \Omega$. Let $C$ be a circle centered at a point of $\mathbb{R}$ with $0 \in C$. It follows from a deep theorem of Solynin [8], that

$$
\lambda(0, \Omega) \geq \lambda\left(0, \mathcal{P}_{C} \Omega\right) .
$$

Moreover, equality holds if and only if either $\Omega=\mathcal{P}_{C} \Omega$ or $\Omega=\mathcal{R}_{C} \mathcal{P}_{C} \Omega$.

2.3. An estimate for the hyperbolic metric. Solynin [9, Theorem 2] proved an estimate for the hyperbolic metric which involves the notion of the ordering of a system of open sets. We will need only a very special case of this estimate; we describe it now: Suppose that $0<m_{1}<1,0<m_{2}<1$, and

$$
\Omega^{*}=\overline{\mathbb{C}} \backslash\left(\left[-1,-1+m_{1}\right] \cup\left[1-m_{2}, 1\right]\right) \text {. }
$$

Then

$$
\lambda^{2}\left(0, \Omega^{*}\right) \geq \lambda\left(0, \Omega_{2}\left(m_{1}\right)\right) \lambda\left(0, \Omega_{2}\left(m_{2}\right)\right)
$$

with equality if and only if $m_{1}=m_{2}$.

\section{Proof of Theorem 3}

First we use an approximation technique. Let

$$
\delta_{1}=\min E_{1}, \quad \eta_{1}=\max E_{1}, \quad \delta_{2}=\min E_{2}, \quad \eta_{2}=\max E_{2} .
$$

The set $\Omega \cap\left(\delta_{1}, \eta_{1}\right)$ is an open subset of the real line. Therefore it is the union of disjoint intervals $\left(a_{j}, b_{j}\right), j=1,2, \ldots$ Similarly, the set $\Omega \cap\left(\delta_{2}, \eta_{2}\right)$ is the union of the disjoint intervals $\left(a_{j}^{\prime}, b_{j}^{\prime}\right), j=1,2, \ldots$ We set

$$
\Omega_{n}=\left(\overline{\mathbb{C}} \backslash\left(\left[\delta_{1}, \eta_{1}\right] \cup\left[\delta_{2}, \eta_{2}\right]\right) \cup \bigcup_{j=1}^{n}\left(\left(a_{j}, b_{j}\right) \cup\left(a_{j}^{\prime}, b_{j}^{\prime}\right)\right), \quad n=1,2, \ldots\right.
$$

The sets $\Omega_{n}$ form an increasing sequence of Denjoy domains, all containing the origin; moreover, $\cup_{n=1}^{\infty} \Omega_{n}=\Omega$. We also note that $\Omega_{n} \rightarrow \Omega$ in the sense of Carathéodory kernel convergence with respect to the origin. By a theorem of D. Hejhal [5],

$$
\lambda\left(0, \Omega_{n}\right) \rightarrow \lambda(0, \Omega) \text { and as } n \rightarrow \infty .
$$

Therefore, in order to prove the inequality $\lambda(0, \Omega) \geq \lambda\left(0, \Omega^{*}\right)$, we may assume that $\overline{\mathbb{C}} \backslash \Omega$ is a union of a finite number $k+m$ of closed intervals:

$$
\overline{\mathbb{C}} \backslash \Omega=\left[t_{1}, t_{2}\right] \cup \cdots \cup\left[t_{k}, t_{k+1}\right] \cup\left[s_{1}, s_{2}\right] \cup \cdots \cup\left[s_{m}, s_{m+1}\right]
$$

with

$$
-1 \leq t_{k+1}<t_{k}<\cdots<t_{2}<t_{1}<0<s_{1}<s_{2}<\cdots<s_{m}<s_{m+1} \leq 1 .
$$

(By the domain monotonicity of the hyperbolic metric, we may ignore isolated points of $\overline{\mathbb{C}} \backslash \Omega$ ). 
After this reduction, we use successive polarizations with respect to suitable circles. Let $C$ be the circle centered at $x>0$ of radius $x$, where $x$ is such that $\mathcal{R}_{C} s_{1}=s_{3}$. A simple computation shows that

$$
x=\frac{s_{1} s_{3}}{s_{1}+s_{3}} .
$$

Then

$$
\mathcal{R}_{C} s_{2}=x+\frac{x^{2}}{s_{2}-x}=\frac{s_{1} s_{2} s_{3}}{s_{1} s_{2}+s_{2} s_{3}-s_{1} s_{3}} .
$$

The reflection in $C$ of the interval $\left[s_{1}, s_{2}\right]$ is the interval $\left[\mathcal{R}_{C} s_{2}, s_{3}\right]$. Moreover, by an elementary simple calculation,

$$
s_{3}-\mathcal{R}_{C} s_{2}>s_{2}-s_{1}
$$

By Solynin's polarization estimate (see subsection 2.2),

$$
\lambda(0, \Omega) \geq \lambda\left(0, G_{1}\right)
$$

where

$$
G_{1}=\overline{\mathbb{C}} \backslash\left(\left[t_{1}, t_{2}\right] \cup \ldots\left[t_{k}, t_{k+1}\right] \cup\left[\mathcal{R}_{C} s_{2}, s_{4}\right] \cup\left[s_{5}, s_{6}\right] \cup \cdots \cup\left[s_{m}, s_{m+1}\right]\right) .
$$

The domain $G_{1}$ is a Denjoy domain containing the origin and also $\left|\overline{\mathbb{C}} \backslash G_{1}\right| \geq$ $|\overline{\mathbb{C}} \backslash \Omega|$. Moreover, $\overline{\mathbb{C}} \backslash G_{1}$ consists of $k+m-1$ intervals.

Continuing this procedure, we find that

$$
\lambda(0, \Omega) \geq \lambda(0, G),
$$

where

$$
\overline{\mathbb{C}} \backslash G=\left[\tau_{1}, \tau_{2}\right] \cup\left[\sigma_{1}, \sigma_{2}\right], \quad-1 \leq \tau_{1}<\tau_{2}<0<\sigma_{1}<\sigma_{2} \leq 1
$$

and $|\overline{\mathbb{C}} \backslash G| \geq|\overline{\mathbb{C}} \backslash \Omega|$. We apply (if necessary) two more polarizations to conclude that

where

$$
\lambda(0, \Omega) \geq \lambda\left(0, \Omega^{\prime}\right)
$$

$$
\Omega^{\prime}=\overline{\mathbb{C}} \backslash\left(\left[-1,-1+m_{1}^{\prime}\right] \cup\left[1-m_{2}^{\prime}, 1\right]\right)
$$

and $m_{1} \leq m_{1}^{\prime}, m_{2} \leq m_{2}^{\prime}$. Note that $\Omega^{\prime} \subset \Omega^{*}$ and hence, by the domain monotonicity of the hyperbolic metric,

$$
\lambda(0, \Omega) \geq \lambda\left(0, \Omega^{*}\right) .
$$

So the inequality (1.4) has been proved.

Now suppose that $\lambda(0, \Omega)=\lambda\left(0, \Omega^{*}\right)$ and that $\Omega \neq \Omega^{*}$. Recall that $E_{1}=$ $(\overline{\mathbb{C}} \backslash \Omega) \cap[-1,0)$ and $E_{2}=(\overline{\mathbb{C}} \backslash \Omega) \cap(0,1]$. Without loss of generality, we may assume that there exists an interval $[\alpha, \beta] \subset \Omega$ with

$$
\min E_{2}=: \beta_{1}<\alpha<\beta<1 \text {. }
$$

Let $C$ be the circle of radius $x_{o}$ centered at $x_{o}$, where

$$
x_{o}=\frac{\beta_{1} \beta}{\beta_{1}+\beta} .
$$


Note that $\mathcal{P}_{C} \beta_{1}=\beta$ and observe that $\Omega \neq \mathcal{P}_{C} \Omega$ and $\Omega \neq \mathcal{R}_{C} \mathcal{P}_{C} \Omega$; otherwise, we would have $E_{1}=\varnothing$ which absurd since $\left|E_{1}\right|=m_{1}>0$. By the sharp form of the polarization estimate (see subsection 2.2) and the first part of Theorem 3 (which we have already proved),

$$
\lambda(0, \Omega)>\lambda\left(0, \mathcal{P}_{C} \Omega\right) \geq \lambda\left(0, \Omega^{*}\right) .
$$

Contradiction. Hence $\Omega=\Omega^{*}$.

\section{Proof of Theorem 2}

In the introduction we defined two classes of domains in $\overline{\mathbb{C}}$ that contain the origin. The simply connected domains

$$
\Omega_{1}(m)=\overline{\mathbb{C}} \backslash[-1,-1+2 m], \quad 0<m<\frac{1}{2}
$$

and the doubly connected domains

$$
\Omega_{2}(m)=\overline{\mathbb{C}} \backslash([-1,-1+m] \cup[1-m, 1]), \quad 0<m<1 .
$$

We will find explicit expressions for the values $\lambda\left(0, \Omega_{1}(m)\right)$ and $\lambda\left(0, \Omega_{2}(m)\right)$.

Fix $m$ with $0<m<\frac{1}{2}$. The function $f_{1}(z)=\frac{2}{m}(z+1)-2$ maps $\Omega_{1}(m)$ conformally onto $\overline{\mathbb{C}} \backslash[-2,2]$ with $f_{1}(0)=2 / m-2$. The inverse of the Joukowski map $J(z)=z+1 / z$ maps $\overline{\mathbb{C}} \backslash[-2,2]$ conformally onto $\overline{\mathbb{C}} \backslash\{|z| \leq 1\}$. Let

$$
b:=J^{-1}\left(\frac{2}{m}-2\right)=\frac{1}{m}-1+\sqrt{\frac{1}{m^{2}}-\frac{2}{m}} .
$$

Finally, the inversion $f_{2}(z)=\frac{1}{z}$ maps $\overline{\mathbb{C}} \backslash\{|z| \leq 1\}$ onto the unit disk $\mathbb{D}$. Therefore the mapping $g=f_{1}^{-1} \circ J \circ f_{2}^{-1}$ maps $\mathbb{D}$ conformally onto $\Omega_{1}(m)$; hence it is a universal covering map of $\Omega_{1}(m)$. By the principle of the hyperbolic metric and by (4.1),

$$
\begin{aligned}
\lambda\left(0, \Omega_{1}(m)\right) & =\frac{\lambda(1 / b, \mathbb{D})}{\left|g^{\prime}(1 / b)\right|} \\
& =\frac{\lambda(1 / b, \mathbb{D})\left|f_{2}^{\prime}(b)\right|\left|f_{1}^{\prime}(0)\right|}{\left|J^{\prime}(b)\right|} \\
& =\frac{\frac{1}{b^{2}} \frac{2}{m}}{\left(1-\frac{1}{b^{2}}\right) \frac{b^{2}-1}{b^{2}}} \\
& =\frac{2 b^{2}}{m\left(b^{2}-1\right)^{2}}=\frac{m}{2-4 m} .
\end{aligned}
$$

Next we find a universal covering map for $\Omega_{2}(m), 0<m<1$. Set $k=1-m$ and note that $0<k<1$. We need the complete elliptic integrals of the first kind,

$$
\mathcal{K}=\mathcal{K}(k):=\int_{0}^{1} \frac{d t}{\sqrt{\left(1-t^{2}\right)\left(1-k^{2} t^{2}\right)}},
$$


and of the second kind

$$
\mathcal{E}=\mathcal{E}(k):=\int_{0}^{1} \sqrt{\frac{1-k^{2} t^{2}}{1-t^{2}}} d t
$$

The basic properties of these functions can be found, for example, in [1, Ch. 3], $[6, \mathrm{Ch} . \mathrm{VI}]$.

The function

$$
z \mapsto \frac{4 \mathcal{K} \tan ^{-1} z}{\pi}
$$

maps $\mathbb{D}$ conformally onto the strip $\{z:-\mathcal{K}<\operatorname{Re} z<\mathcal{K}\}$. The Jacobian elliptic sine function $\operatorname{sn} z=\operatorname{sn}(z, k)$ maps this strip onto $\overline{\mathbb{C}} \backslash([-1 / k,-1] \cup[1,1 / k])$; see [6]. Finally, the map $z \mapsto k z$ maps $\overline{\mathbb{C}} \backslash([-1 / k,-1] \cup[1,1 / k])$ onto $\overline{\mathbb{C}} \backslash([-1,-1+$ $m] \cup[1-m, 1])$; recall that $m=1-k$. Therefore the function

$$
h(z)=k \operatorname{sn}\left(\frac{4 \mathcal{K}(k) \tan ^{-1} z}{\pi}\right)
$$

maps $\mathbb{D}$ onto $\Omega_{2}(m)$. Moreover, it follows from the construction of the elliptic sine function via the reflection principle (see [6]) that every function element of $h^{-1}$ can be analytically continued throughout $\Omega_{2}(m)$ in such a way that all of its values remain in $\mathbb{D}$. In the terminology of Hayman [4, Ch. 9] this means that $h$ maps $\mathbb{D}$ onto $\Omega_{2}(m)$. In other wards, $h$ is a universal covering map of $\Omega_{2}(m)$. By the definition of the hyperbolic metric, the fact that $h(0)=0$, and some formulas for the Jacobi elliptic functions (see [1, Ch. 6]),

$$
\lambda\left(0, \Omega_{2}(m)\right)=\frac{1}{h^{\prime}(0)}=\frac{\pi}{4 k \mathcal{K}(k)}=\frac{\pi}{4(1-m) \mathcal{K}(1-m)} .
$$

We now compare $\lambda\left(0, \Omega_{1}(m)\right)$ with $\lambda\left(0, \Omega_{2}(m)\right)$. Let

$$
g_{1}(m)=\frac{\lambda\left(0, \Omega_{2}(m)\right)}{\lambda\left(0, \Omega_{1}(m)\right)}=\frac{\pi(1-2 m)}{2 m(1-m) \mathcal{K}(1-m)}, \quad 0<m<\frac{1}{2} .
$$

We will prove that $g_{1}$ is strictly decreasing. It suffices to prove that the function

$$
g_{2}(k)=\frac{2 k-1}{k(1-k) \mathcal{K}(k)}, \quad \frac{1}{2}<k<1,
$$

is strictly increasing. To prove that, we will use some formulas and inequalities for complete elliptic integrals taken from [1, Ch. 3]. First differentiate to get

$$
g_{2}^{\prime}(k)=\frac{k(1+k) \mathcal{K}(k)-(2 k-1) \mathcal{E}(k)}{(1-k)^{2} k^{2}(1+k) \mathcal{K}^{2}(k)} .
$$

So it suffices to show that

$$
\frac{\mathcal{K}(k)}{\mathcal{E}(k)} \geq \frac{2 k-1}{k(k+1)}, \quad \frac{1}{2}<k<1 .
$$

By [1, Exercise 3.43(34)], the function

$$
\frac{\mathcal{K}(k)}{\mathcal{E}(k)}-\log \frac{4}{\sqrt{1-k^{2}}}
$$


is increasing for $k \in(0,1)$. Hence for $k \in(1 / 2,1)$, we have

$$
\frac{\mathcal{K}(k)}{\mathcal{E}(k)} \geq \frac{\mathcal{K}(1 / 2)}{\mathcal{E}(1 / 2)}-\log \frac{4}{\sqrt{1-(1 / 2)^{2}}}+\log \frac{4}{\sqrt{1-k^{2}}}>\log \frac{4}{\sqrt{1-k^{2}}}-0.4 .
$$

Therefore, in order to prove (4.4), it suffices to show that

$$
\log \frac{4}{\sqrt{1-k^{2}}}-0.4 \geq \frac{2 k-1}{k(k+1)}, \quad k \in(1 / 2,1) .
$$

The inequality (4.5) can be easily proved by elementary calculus.

So we have proved that the function $g_{1}$ is strictly decreasing for $m \in(0,1 / 2)$. Therefore, there exists a unique number $m^{\sharp}$ such that:

For $m>m^{\sharp}, g_{1}(m)<1$ and hence $\lambda\left(0, \Omega_{1}(m)\right)>\lambda\left(0, \Omega_{2}(m)\right)$;

for $m=m^{\sharp}, g_{1}(m)=1$ and hence $\lambda\left(0, \Omega_{1}(m)\right)=\lambda\left(0, \Omega_{2}(m)\right)$;

for $m<m^{\sharp}, g_{1}(m)>1$ and hence $\lambda\left(0, \Omega_{1}(m)\right)<\lambda\left(0, \Omega_{2}(m)\right)$.

We finally observe that $g_{1}(0.3684)>1$ and $g_{1}(0.3685)<1$. Hence

$$
0.3684<m^{\sharp}<0.3685 \text {. }
$$

The proof of Theorem 2 is now complete.

\section{Proof of Theorem 1 and Corollary 1}

We need some lemmas for complete elliptic integrals.

Lemma 1. For $0<k<1$,

$$
\left(1-3 k^{2}\right) \mathcal{E}^{2}(k)<2 k^{2}\left(1-k^{2}\right) \mathcal{E}(k) \mathcal{K}(k)+\left(1-k^{2}\right)^{2} \mathcal{K}^{2}(k) .
$$

Proof. By the obvious inequality $\mathcal{E} \leq \mathcal{K}, k \in(0,1)$, it suffices to show that

$$
\left(1-3 k^{2}\right)<2 k^{2}\left(1-k^{2}\right)+\left(1-k^{2}\right)^{2} .
$$

This inequality is equivalent to $k^{2}<3$ which is true.

Lemma 2. There exists a number $m^{*}, 0.31861<m^{*}<0.31862$, with the property: For $m \in\left[m^{*}, 1\right]$,

$$
\mathcal{E}^{2}(1-m)-\left(2-2 m+m^{2}\right) \mathcal{E}(1-m) \mathcal{K}(1-m)+(2-m) m \mathcal{K}^{2}(1-m) \geq 0
$$

with equality if and only if $m=m^{*}$.

Proof. For $m \in(0,1]$, we set

$$
F(m)=\mathcal{E}^{2}(1-m)-\left(2-2 m+m^{2}\right) \mathcal{E}(1-m) \mathcal{K}(1-m)+(2-m) m \mathcal{K}^{2}(1-m) .
$$

Using the formulas for the derivatives of $\mathcal{K}, \mathcal{E}$ (see $[1$, Ch. 3]), we see that $F^{\prime}(m)>0$ if and only if

$$
\begin{array}{r}
\left(2-6 m+3 m^{2}\right) \mathcal{E}^{2}(1-m)+2 m(2-m)(1-m)^{2} \mathcal{E}(1-m) \mathcal{K}(1-m) \\
+(2-m)^{2} m^{2} \mathcal{K}^{2}(1-m)>0 .
\end{array}
$$

If we set $k=1-m$, the above condition becomes

$$
\left(1-3 k^{2}\right) \mathcal{E}^{2}(k)<2 k^{2}\left(1-k^{2}\right) \mathcal{E}(k) \mathcal{K}(k)+\left(1-k^{2}\right)^{2} \mathcal{K}^{2}(k)
$$


which is true, by Lemma 1 . Hence the function $F$ is strictly increasing for $m \in$ $(0,1]$. Therefore there exists a unique number $m^{*} \in(0,1)$ such that $F\left(m^{*}\right)=0$ and $F(m)>0$ for $m \in\left(m^{*}, 1\right]$. Since $F(0.31861)<0$ and $F(0.31862)>0$, we have $0.31861<m^{*}<0.31862$.

Lemma 3. The function

$$
h(m)=\log \frac{\pi}{4(1-m) \mathcal{K}(1-m)}, \quad m \in\left[m^{*}, 1\right]
$$

is convex.

Proof. A simple computation involving the derivatives of $\mathcal{K}, \mathcal{E}$ shows that $h^{\prime \prime}(m) \geq$ 0 if and only if $F(m) \geq 0$. It follows from Lemma 2 that $h$ is convex in $\left[m^{*}, 1\right]$.

\section{Proof of Theorem 1}

Let $m^{*}$ be the number appearing in Lemma 3. Suppose that $m^{*} \leq m_{1}<1$, $m^{*} \leq m_{2}<1$ and consider a domain

$$
\Omega=\overline{\mathbb{C}} \backslash\left(E_{1} \cup E_{2}\right)
$$

where

$$
E_{1} \subset[-1,0),\left|E_{1}\right|=m_{1}, \quad E_{2} \subset(0,1],\left|E_{2}\right|=m_{2} .
$$

By Theorem 3,

$$
\lambda(0, \Omega) \geq \lambda\left(0, \Omega^{*}\right)
$$

where

$$
\Omega^{*}=\overline{\mathbb{C}} \backslash\left(\left[-1,-1+m_{1}\right] \cup\left[1-m_{2}, 1\right]\right) .
$$

By Solynin's inequality (2.1),

$$
\lambda^{2}\left(0, \Omega^{*}\right) \geq \lambda\left(0, \Omega_{2}\left(m_{1}\right)\right) \lambda\left(0, \Omega_{2}\left(m_{2}\right)\right) .
$$

By (4.3) and Lemma 3, the function

$$
h(m)=\log \lambda\left(0, \Omega_{2}(m)\right)
$$

is convex for $m \in\left[m^{*}, 1\right]$. Hence

$$
\lambda\left(0, \Omega_{2}\left(m_{1}\right)\right) \lambda\left(0, \Omega_{2}\left(m_{2}\right)\right) \geq \lambda^{2}\left(0, \Omega_{2}\left(\frac{m_{1}+m_{2}}{2}\right)\right) .
$$

Combining the inequalities (5.1), (5.2), (5.3), we conclude that

$$
\lambda(0, \Omega) \geq \lambda\left(0, \Omega_{2}\left(\frac{m_{1}+m_{2}}{2}\right)\right)
$$

which is (1.3).

Suppose that equality holds in (1.3). Then equality holds in both (5.1) and (5.2). Equality in (5.1) implies that $\Omega=\Omega^{*}$; see Theorem 3. Also equality in (5.2) implies that $m_{1}=m_{2}=\left(m_{1}+m_{2}\right) / 2$, namely $\Omega^{*}=\Omega_{2}\left(\frac{m_{1}+m_{2}}{2}\right)$.

\section{Proof of Corollary 1}

Let

$$
\Omega=\overline{\mathbb{C}} \backslash E, \quad E \subset[-1,1] \backslash\{0\},|E|=2 m
$$


and suppose that $m \geq \frac{1+m^{*}}{2}$. Let

$$
E_{1}:=E \cap[-1,0), \quad E_{2}:=E \cap(0,1] .
$$

Then $m_{1}:=\left|E_{1}\right| \geq m^{*}$ and $m_{2}:=\left|E_{2}\right| \geq m^{*}$. So we may apply Theorem 1 to conclude that

$$
\lambda(0, \Omega) \geq \lambda\left(0, \Omega_{2}(m)\right)
$$

with equality if and only if $\left.\Omega=\Omega_{2}\left(\frac{m_{1}+m_{2}}{2}\right)\right)=\Omega_{2}(m)$.

\section{REFERENCES}

1. G.D. Anderson, M.K. Vamanamurthy, and M. Vuorinen, Conformal Invariants, Inequalities, and Quasiconformal Maps, Wiley, 1997.

2. A.F. Beardon, D. Minda, The hyperbolic metric and geometric function theory, Proceedings of the International Workshop on Quasiconformal Mappings and their Applications, Narosa Publishing House, India, (2006), 159-206.

3. G.M. Goluzin, Geometric Theory of Functions of a Complex Variable, Amer. Math. Soc., 1969.

4. W.K. Hayman, Subharmonic Functions, vol.2. Academic Press, 1989.

5. D. Hejhal, Universal covering maps for variable regions, Math. Z., 137 (1974), 7-20.

6. Z. Nehari, Conformal Mapping. Reprinting of the 1952 edition. Dover Publications, 1975.

7. R. Nevanlinna, Analytic Functions. Springer, 1970.

8. A.Yu. Solynin, Functional inequalities via polarization, St.Petersburg Math. J. 8 (1997), 1015-1038 (translated from Algebra i Analiz 8 (1996), 148-185).

9. A.Yu. Solynin, Ordering of sets, hyperbolic metrics, and harmonic measures, J. Math. Sci. (New York) 95 (1999), 2256-2266 (translated from Zap. Nauchn. Sem. (POMI) 237 (1997), 129-147).

10. A.Yu. Solynin, Radial projection and the Poincaré metric, J. Math. Sci. (New York) 95 (1999), 2267-2275 (translated from Zap. Nauchn. Sem. (POMI) 237 (1997), 148-160).

11. A.Yu. Solynin, M. Vuorinen, Estimates for the hyperbolic metric of the punctured plane and applications, Israel J. Math. 124 (2001), 29-60.

Department of Mathematics, Aristotle University of Thessaloniki, 54124 ThesSALONIKI, GREECE

E-mail address: betsakos@math.auth.gr 